TRANSACTIONS OF THE

AMERICAN MATHEMATICAL SOCIETY

Volume 365, Number 10, October 2013, Pages 5237-5257

S 0002-9947(2013)05799-6

Article electronically published on March 5, 2013

\title{
THE PROBABILITY DISTRIBUTIONS OF THE FIRST HITTING TIMES OF BESSEL PROCESSES
}

\author{
YUJI HAMANA AND HIROYUKI MATSUMOTO
}

\begin{abstract}
We consider the first hitting times of the Bessel processes. We give explicit expressions for the distribution functions by means of the zeros of the Bessel functions. The resulting formula is simpler and easier to treat than the corresponding results which have already been obtained.
\end{abstract}

\section{INTRODUCTION}

In this article we consider the first hitting time of the Bessel process, which itself is an interesting object and is one of the important tools to study several problems in probability theory. By general theory of one-dimensional diffusion processes, the Laplace transform of the distribution satisfies an eigenvalue problem for the generator and it is given by a ratio of the modified Bessel functions.

Except for some special cases, it is not easy to invert the Laplace transforms. When the index $\nu$ of the Bessel process is a half integer $n+1 / 2, n \in \mathbb{N}$, the Macdonald function $K_{\nu}$ is of a simple form. In this case, it turns out that $K_{\nu+1} / K_{\nu}$ is represented by the ratio of polynomials. With the help of the partial fraction decomposition, Hamana 9], 10] recently has inverted the Laplace transform and applied the results to show the explicit form and the asymptotic behavior of the expected volume of the Wiener sausage for the odd dimensional Brownian motion. The method used in [10] requires some formulae for the zeros of $K_{\nu}$.

When the starting point of a Bessel process is closer to the boundary 0 than its arrival point, the Laplace transform of the first hitting time is given by a ratio of the modified Bessel function $I_{\nu}$. In this case, Kent [14] has given an explicit expression (see $(2.7),(2,8)$ and $(2.9)$ below) by means of the zeros of $I_{\nu}$ or the Bessel function $J_{\nu}$ for the density in his general framework.

The purpose of this paper is to show the explicit formula for the distribution function in the other case, where the boundary $\infty$ is natural and the Laplace transform is written by a ratio of $K_{\nu}$ 's. For our expressions, we need the zeros of $K_{\nu}$. In order to prove the results, we represent the ratio of the Macdonald functions by using contour integrals of functions easier to treat, and invert the Laplace transform. Recently Byczkowski, et al. [2, 3] have given similar but different expressions for the densities of the first hitting times, and applied the results to some study on

Received by the editors June 30, 2011 and, in revised form, July 26, 2011 and January 24, 2012 .

2010 Mathematics Subject Classification. Primary 60J60; Secondary 33C10, 44A10.

Key words and phrases. Bessel process, first hitting time, Bessel functions.

The authors were partially supported by the Grant-in-Aid for Scientific Research (C) No. 20540121 and 23540183, Japan Society for the Promotion of Science. 
geometric Brownian motions and hyperbolic Brownian motions. We use the same curve for the contour integral. However, we show a decomposition of the Bessel function ratio and use it, which makes our expression simpler.

Moreover we should mention that Ismail has considered several types of functions given as the ratios of modified Bessel functions and discussed when the functions are completely monotone; see [11 and the references therein. In particular he showed that the function on the right hand side of (2.5) below is completely monotone by expressing it as a Stieltjes transform of some function. This result gives another expression for the distribution function of the first hitting time, while it is more complicated.

Ismail has also shown that the function $K_{\nu+1} / K_{\nu}$ is completely monotone in a similar way. In our context, such a function appears if we give an expression for the Lévy measure of the distribution of the first hitting time. The Lévy measure will be deduced in the forthcoming paper. General theory on the infinite divisibility of the distributions of the first hitting times of one-dimensional diffusion processes is given by Yamazato [19].

This article is organized as follows. We give the main result, Theorem 2.2, in Section 2 and prove it in Section 3. Section 4 is devoted to the asymptotic behavior of the tail probability of the first hitting time, which is obtained as an application of the result.

\section{The First hitting time of the Bessel Processes}

For $\nu \in \mathbb{R}$ the one-dimensional diffusion process with infinitesimal generator

$$
\mathcal{G}^{(\nu)}=\frac{1}{2} \frac{d^{2}}{d x^{2}}+\frac{2 \nu+1}{2 x} \frac{d}{d x}=\frac{1}{2 x^{2 \nu+1}} \frac{d}{d x}\left(x^{2 \nu+1} \frac{d}{d x}\right)
$$

is called the Bessel process with index $\nu$. If $2 \nu+2$ is a positive integer, the Bessel process is identical in law with the radial motion of a $(2 \nu+2)$-dimensional Brownian motion. Hence, $2 \nu+2$ is called the dimension of the Bessel process.

The classification of boundary points gives the following information. The endpoint $\infty$ is a natural boundary for any $\nu \in \mathbb{R}$. For $\nu \geqq 0,0$ is an entrance and not exit boundary. For $-1<\nu<0$, 0 is a regular boundary, which is instantly reflecting. For $\nu \leqq-1,0$ is an exit but not entrance boundary. For more details, see 12 and [17].

For $a, b \geqq 0$ we denote by $\tau_{a, b}^{(\nu)}$ the first hitting time to $b$ of the Bessel process with index $\nu$ starting at $a$. By general theory of one-dimensional diffusion processes, we can evaluate the Laplace transform of the distribution of $\tau_{a, b}^{(\nu)}$ by solving an eigenvalue problem. In fact, denoting by $E$ the expectation, we have that the function

$$
x \mapsto E\left[e^{-\lambda \tau_{x, b}^{(\nu)}}\right]
$$

is increasing (decreasing) on $[0, b)$ (resp. $(b, \infty))$ and satisfies

$$
\mathcal{G}^{(\nu)} u=\lambda u, \quad u(b)=1 .
$$

The following explicit expressions for $E\left[e^{-\lambda \tau_{a, b}^{(\nu)}}\right]$ are well known (cf. [7], [13]): for $\lambda>0$, if $b>0$ and $\nu>-1$,

$$
E\left[e^{-\lambda \tau_{0, b}^{(\nu)}}\right]=\frac{(b \sqrt{2 \lambda})^{\nu}}{2^{\nu} \Gamma(\nu+1)} \frac{1}{I_{\nu}(b \sqrt{2 \lambda})} ;
$$


if $0<a \leqq b$ and $\nu>-1$,

$$
E\left[e^{-\lambda \tau_{a, b}^{(\nu)}}\right]=\frac{a^{-\nu} I_{\nu}(a \sqrt{2 \lambda})}{b^{-\nu} I_{\nu}(b \sqrt{2 \lambda})}
$$

if $0<a \leqq b$ and $\nu \leqq-1$,

$$
E\left[e^{-\lambda \tau_{a, b}^{(\nu)}}\right]=\frac{a^{-\nu} I_{-\nu}(a \sqrt{2 \lambda})}{b^{-\nu} I_{-\nu}(b \sqrt{2 \lambda})}
$$

if $a>0$ and $\nu<0$,

$$
E\left[e^{-\lambda \tau_{a, 0}^{(\nu)}}\right]=\frac{2^{\nu+1}}{\Gamma(|\nu|)(a \sqrt{2 \lambda})^{\nu}} K_{\nu}(a \sqrt{2 \lambda})
$$

if $0<b \leqq a$ and $\nu \in \mathbb{R}$,

$$
E\left[e^{-\lambda \tau_{a, b}^{(\nu)}}\right]=\frac{a^{-\nu} K_{\nu}(a \sqrt{2 \lambda})}{b^{-\nu} K_{\nu}(b \sqrt{2 \lambda})} .
$$

Here $\Gamma$ is the gamma function and $I_{\nu}$ and $K_{\nu}$ denote modified Bessel functions of the first and the second kinds of order $\nu$, respectively. Both $I_{\nu}$ and $K_{\nu}$ are the solutions of the modified Bessel differential equation

$$
z^{2} \frac{d^{2} w}{d z^{2}}+z \frac{d w}{d z}-\left(z^{2}+\nu^{2}\right) w=0 .
$$

The distribution function of $\tau_{a, b}^{(\nu)}$ for $a<b$ was obtained in [14. If $b>0$ and $\nu>-1$,

$$
P\left(\tau_{0, b}^{(\nu)} \leqq t\right)=1-\frac{1}{2^{\nu-1} \Gamma(\nu+1)} \sum_{k=1}^{\infty} \frac{j_{\nu, k}^{\nu-1}}{J_{\nu+1}\left(j_{\nu, k}\right)} e^{-\frac{j_{\nu, k}^{2}}{2 b^{2}} t} .
$$

If $0<a<b$, we have that, $\nu>-1$,

$$
P\left(\tau_{a, b}^{(\nu)} \leqq t\right)=1-2\left(\frac{b}{a}\right)^{\nu} \sum_{k=1}^{\infty} \frac{J_{\nu}\left(a j_{\nu, k} / b\right)}{j_{\nu, k} J_{\nu+1}\left(j_{\nu, k}\right)} e^{-\frac{j_{\nu, k}^{2}}{2 b^{2}} t}
$$

and that, for $\nu \leqq-1$,

$$
P\left(\tau_{a, b}^{(\nu)} \leqq t\right)=\left(\frac{b}{a}\right)^{2 \nu}-2\left(\frac{b}{a}\right)^{\nu} \sum_{k=1}^{\infty} \frac{J_{-\nu}\left(a j_{-\nu, k} / b\right)}{j_{-\nu, k} J_{-\nu+1}\left(j_{-\nu, k}\right)} e^{-\frac{j_{-\nu, k}^{2}}{2 b^{2}} t} .
$$

Here $J_{\mu}$ is the Bessel function of the first kind of order $\mu$ and $\left\{j_{\nu, k}\right\}_{k=1}^{\infty}$ is the increasing sequence of positive zeros of $J_{\nu}$.

When $b>0$ and $2 \nu+2$ is a positive integer, Ciesielski and Taylor 4 have already shown (2.7). When $2 \nu+2$ is not a positive integer, it is possible to derive the inverse Laplace transform of the right hand side of (2.1) by the same methods as those used to prove Theorem 1 in [4]. The formula (2.8) immediately shows that, if $0<a<b$ and $\nu>-1$,

$$
P\left(\tau_{a, b}^{(\nu)}>t\right)=2\left(\frac{b}{a}\right)^{\nu} \frac{J_{\nu}\left(a j_{\nu, 1} / b\right)}{j_{\nu, 1} J_{\nu+1}\left(j_{\nu, 1}\right)} e^{-\frac{j_{\nu, 1}^{2}}{2 b^{2}} t}\{1+o(1)\} .
$$

A similar asymptotic result, in the case where $a=0$ and $2 \nu+2$ is an integer greater than 2 (Brownian case), was used in [4] to show the law of iterated logarithm for the total time spent by the Bessel process in $(0, b)$ as $b \downarrow 0$. 
Remark 2.1. We find in [5], p. 104, the formula

$$
\frac{J_{\nu}(c z)}{J_{\nu}(z)}=c^{\nu}+\sum_{k=1}^{\infty} \frac{2 z^{2}}{j_{\nu, k}\left(j_{\nu, k}^{2}-z^{2}\right)} \frac{J_{\nu}\left(c j_{\nu, k}\right)}{J_{\nu+1}\left(j_{\nu, k}\right)}
$$

for $0 \leqq c \leqq 1$, which implies (2.8) and (2.9) by virtue of $I_{\nu}(x)=e^{-i \pi \nu / 2} J_{\nu}\left(x e^{i \pi / 2}\right)$ for $x>0$ (cf. [18, p. 77).

In the case $a>0$ and $\nu<0$, we can easily check

$$
P\left(\tau_{a, 0}^{(\nu)} \leqq t\right)=\frac{2^{\nu}}{\Gamma(|\nu|) a^{2}} \int_{0}^{t} s^{\nu-1} e^{-\frac{a^{2}}{2 s}},
$$

by (2.4) and the formula

$$
K_{\nu}(z)=\frac{1}{2}\left(\frac{z}{2}\right)^{\nu} \int_{0}^{\infty} e^{-t-\frac{z^{2}}{4 t}} t^{-\nu-1} d t .
$$

To give our result on the distribution functions of $\tau_{a, b}^{(\nu)}$ in the case of $0<b<a$, we need to recall some facts about the zeros of the Bessel function $K_{\nu}$. For $\nu \in \mathbb{R}$ we denote by $N(\nu)$ the number of zeros of $K_{\nu}$. It is known that $N(\nu)=|\nu|-1 / 2$ if $\nu-1 / 2$ is an integer and that $N(\nu)$ is the even number closest to $|\nu|-1 / 2$ otherwise. We remark that $N(\nu)=0$ if $|\nu|<3 / 2$ and $N(\nu) \geqq 1$ if $|\nu| \geqq 3 / 2$. Each zero, if it exists, lies in the half plane $\{z \in \mathbb{C} ; \operatorname{Re}(z)<0\}$, denoted by $\mathbb{C}^{-}$. In this case, we write $z_{\nu, 1}, z_{\nu, 2}, \ldots, z_{\nu, N(\nu)}$ for the zeros. Since $K_{\nu}$ is a solution of $(2.6)$, all zeros of $K_{\nu}$ are of multiplicity one by the uniqueness of the solution of ordinary differential equations. This means that all zeros of $K_{\nu}$ are distinct. If $\nu-1 / 2$ is not an integer, there are no real zeros. For details, see [18], pp. 511-513.

Theorem 2.2. Let $0<b<a$. For $\mu \geqq 0$ and $c>1$, we set

$$
L_{\mu, c}(x)=\frac{\cos (\pi \mu)\left\{I_{\mu}(c x) K_{\mu}(x)-I_{\mu}(x) K_{\mu}(c x)\right\}}{\left\{K_{\mu}(x)\right\}^{2}+\pi^{2}\left\{I_{\mu}(x)\right\}^{2}+2 \pi \sin (\pi \mu) K_{\mu}(x) I_{\mu}(x)} .
$$

(1) If $\nu= \pm 1 / 2$,

$$
P\left(\tau_{a, b}^{(\nu)} \leqq t\right)=\left(\frac{b}{a}\right)^{\nu+|\nu|} \int_{0}^{t} \frac{a-b}{\sqrt{2 \pi s^{3}}} e^{-\frac{(a-b)^{2}}{2 s}} d s .
$$

(2) If $|\nu|<3 / 2$ and $\nu \neq \pm 1 / 2$,

$$
\begin{aligned}
& P\left(\tau_{a, b}^{(\nu)} \leqq t\right)=\left(\frac{b}{a}\right)^{\nu+|\nu|} \int_{0}^{t} \frac{a-b}{\sqrt{2 \pi s^{3}}} e^{-\frac{(a-b)^{2}}{2 s}} d s \\
& -\left(\frac{b}{a}\right)^{\nu} \int_{0}^{t} \frac{a-b}{\sqrt{2 \pi s^{3}}} e^{-\frac{(a-b)^{2}}{2 s}}\left[\int_{0}^{\infty} \frac{L_{|\nu|, a / b}(x)}{x} e^{-\frac{x(a-b) \sqrt{t}}{b \sqrt{s}}} d x\right] d s .
\end{aligned}
$$

(3) If $\nu-1 / 2$ is an integer and $\nu \neq \pm 1 / 2$,

$$
\begin{aligned}
P\left(\tau_{a, b}^{(\nu)} \leqq t\right)= & \left(\frac{b}{a}\right)^{\nu+|\nu|} \int_{0}^{t} \frac{a-b}{\sqrt{2 \pi s^{3}}} e^{-\frac{(a-b)^{2}}{2 s}} d s \\
& -\left(\frac{b}{a}\right)^{\nu} \sum_{j=1}^{N(\nu)} \frac{K_{\nu}\left(a z_{\nu, j} / b\right)}{z_{\nu, j} K_{\nu+1}\left(z_{\nu, j}\right)} \int_{0}^{t} \frac{a-b}{\sqrt{2 \pi s^{3}}} e^{-\frac{(a-b)^{2}}{2 s}+\frac{z_{\nu, j}(a-b) \sqrt{t}}{b \sqrt{s}}} d s .
\end{aligned}
$$


(4) If $\nu-1 / 2$ is not an integer and $|\nu|>3 / 2$,

$$
\begin{aligned}
P\left(\tau_{a, b}^{(\nu)} \leqq t\right)= & \left(\frac{b}{a}\right)^{\nu+|\nu|} \int_{0}^{t} \frac{a-b}{\sqrt{2 \pi s^{3}}} e^{-\frac{(a-b)^{2}}{2 s}} d s \\
& -\left(\frac{b}{a}\right)^{\nu} \sum_{j=1}^{N(\nu)} \frac{K_{\nu}\left(a z_{\nu, j} / b\right)}{z_{\nu, j} K_{\nu+1}\left(z_{\nu, j}\right)} \int_{0}^{t} \frac{a-b}{\sqrt{2 \pi s^{3}}} e^{-\frac{(a-b)^{2}}{2 s}+\frac{z_{\nu, j}(a-b) \sqrt{t}}{b \sqrt{s}}} d s \\
& -\left(\frac{b}{a}\right)^{\nu} \int_{0}^{t} \frac{a-b}{\sqrt{2 \pi s^{3}}} e^{-\frac{(a-b)^{2}}{2 s}}\left[\int_{0}^{\infty} \frac{L_{|\nu|, a / b}(x)}{x} e^{-\frac{x(a-b) \sqrt{t}}{b \sqrt{s}}} d x\right] d s .
\end{aligned}
$$

From this theorem we can deduce the asymptotic behavior of $P\left(\tau_{a, b}^{(\nu)}>t\right)$ for $0<b<a$, which will be discussed in Section 4. It should be mentioned that, when $\nu=0$, it is obtained from (2.5). In fact, we have

$$
\begin{aligned}
\int_{0}^{\infty} e^{-\lambda t} P\left(\tau_{a, b}^{(\nu)}>t\right) d t & =\frac{1}{\lambda} \frac{K_{0}(b \sqrt{2 \lambda})-K_{0}(a \sqrt{2 \lambda})}{K_{0}(b \sqrt{2 \lambda})} \\
& =\frac{2 \log (a / b)}{\lambda \log (1 / \lambda)}\{1+o(1)\}
\end{aligned}
$$

as $\lambda \rightarrow 0$. The Tauberian theorem of the exponential type immediately yields

$$
P\left(\tau_{a, b}^{(\nu)}>t\right)=\frac{2 \log (a / b)}{\log t}+o\left(\frac{1}{\log t}\right)
$$

as $t \rightarrow \infty$ (cf. [6], p. 446). In order to derive the second equality of (2.10), we have applied the asymptotic behavior of $K_{0}(x)$ as $x \downarrow 0$ (cf. (3.15)). We can directly deduce (2.11) from Theorem 2.2 without the Tauberian theorem, however, the calculation is left to the reader.

In the case of $\nu \neq 0$, we cannot obtain a convenient formula such as (2.10) which allows us to apply the Tauberian theorem in a straightforward way.

\section{The Distribution FUnCTION OF The FiRst HitTing TIME}

From now on, for a suitable function $f$, the notation $\mathcal{L}[f]$ implies the Laplace transform of $f$ and the inverse Laplace transform of $f$ is denoted by $\mathcal{L}^{-1}[f]$. All formulae concerning Laplace and inverse Laplace transforms can be found in [16].

This section is devoted to a proof of Theorem 2.2. For $t>0$ and $\nu \in \mathbb{R}$ let

$$
F_{a, b}^{(\nu)}(t)=P\left(\tau_{a, b}^{(\nu)} \leqq t\right) .
$$

A standard formula shows that

$$
\mathcal{L}\left[F_{a, b}^{(\nu)}\right](\lambda)=\frac{1}{\lambda} E\left[e^{\lambda \tau_{a, b}^{(\nu)}}\right]
$$

for $\lambda>0$. For simplicity we put $G_{a, b}^{(\nu)}(t)=F_{a, b}^{(\nu)}\left(2 b^{2} t\right)$. Then we have

$$
\mathcal{L}\left[G_{a, b}^{(\nu)}\right](\lambda)=\frac{1}{2 b^{2}} \mathcal{L}\left[F_{a, b}^{(\nu)}\right]\left(\frac{\lambda}{2 b^{2}}\right) .
$$

Set $\alpha=a / b>1$. It follows from (2.5) that, for $\lambda>0$,

$$
\mathcal{L}\left[G_{a, b}^{(\nu)}\right](\lambda)=\frac{1}{\alpha^{\nu}} \frac{K_{\nu}(\alpha \sqrt{\lambda})}{\lambda K_{\nu}(\sqrt{\lambda})} .
$$

Since $K_{\nu}=K_{-\nu}$ for $\nu \geqq 0$, it is sufficient to consider the case where $\nu \geqq 0$. 
Let $\nu \geqq 0$ and $c>1$. We first assume that $\nu-1 / 2$ is an integer. In this case, there is a suitable polynomial $\psi_{\nu}$ of order $\nu-1 / 2$ on $\mathbb{C}$ such that $\psi_{\nu}(0) \neq 0$ and

$$
z^{\nu} K_{\nu}(z)=\sqrt{\frac{\pi}{2}} e^{-z} \psi_{\nu}(z)
$$

for $z \in D$ (cf. 15, [18]). Here the notation $D$ has been used to denote the set of points $z \in \mathbb{C} \backslash\{0\}$ with $|\arg z|<\pi$. For example,

$$
\psi_{1 / 2}(z)=1, \quad \psi_{3 / 2}(z)=1+z, \quad \psi_{5 / 2}(z)=3+3 z+z^{2} .
$$

The function $z^{\nu} K_{\nu}(z)$ is extended to an entire function and all zeros of $\psi_{\nu}$ are the same as those of $K_{\nu}$. For $z \in \mathbb{C}$ let

$$
\psi_{\nu, c}(z)=\frac{e^{-(c-1) z} \psi_{\nu}(c z)}{c^{\nu} \psi_{\nu}(z)} .
$$

Then $\psi_{\nu, c}$ is a single-valued meromorphic function on $\mathbb{C}$ and it holds that

$$
\psi_{\nu, c}(z)=\frac{K_{\nu}(c z)}{K_{\nu}(z)}
$$

for $z \in D$. Therefore, if $z \in \mathbb{C}$ is not a zero of $K_{\nu}$, we have

$$
\psi_{\nu, c}(z)=\lim _{v \rightarrow z} \frac{K_{\nu}(c v)}{K_{\nu}(v)}
$$

which implies that $K_{\nu}(c x) / K_{\nu}(x)$ can be determined uniquely for $x<0$ if $x$ is not a zero of $K_{\nu}$.

Recall our notation $z_{\nu, 1}, \ldots, z_{\nu, N(\nu)}$ for the zeros of $K_{\nu}$. Let $w$ be a point in $D$ with $K_{\nu}(w) \neq 0$. We take $R$ so large that $w$ and all the zeros of $K_{\nu}$ are inside $C(R)$, a circle whose center is the origin and radius $\mathrm{R}$.

We set

$$
\Theta(R)=\frac{1}{2 \pi i} \int_{C(R)} g_{\nu, c}^{w}(z) d z
$$

where

$$
g_{\nu, c}^{w}(z)=\frac{w e^{(c-1) z} \psi_{\nu, c}(z)}{z(z-w)}
$$

for $z \in \mathbb{C}$. The singular points of $g_{\nu, c}^{w}$ are $0, w$ and the zeros of $K_{\nu}$, which are all poles of order 1 . The residue theorem yields that, if $N(\nu)=0$,

$$
\Theta(R)=\operatorname{Res}\left(0 ; g_{\nu, c}^{w}\right)+\operatorname{Res}\left(w ; g_{\nu, c}^{w}\right)
$$

and that, if $N(\nu) \geqq 1$,

$$
\Theta(R)=\operatorname{Res}\left(0 ; g_{\nu, c}^{w}\right)+\operatorname{Res}\left(w ; g_{\nu, c}^{w}\right)+\sum_{j=1}^{N(\nu)} \operatorname{Res}\left(z_{\nu, j} ; g_{\nu, c}^{w}\right),
$$

where $\operatorname{Res}(v ; f)$ is the residue of a function $f$ at a pole $v$. By definition of the function $g_{\nu, c}^{w}$, we have

$$
\operatorname{Res}\left(0 ; g_{\nu, c}^{w}\right)=-\psi_{\nu, c}(0)=-\frac{1}{c^{\nu}}
$$

and

$$
\operatorname{Res}\left(w ; g_{\nu, c}^{w}\right)=e^{(c-1) w} \psi_{\nu, c}(w)=e^{(c-1) w} \frac{K_{\nu}(c w)}{K_{\nu}(w)}
$$


If $N(\nu) \geqq 1$, the residue of $g_{\nu, c}^{w}$ at $z_{\nu, j}$ is equal to

$$
\lim _{z \rightarrow z_{\nu, j}} \frac{w \psi_{\nu}(c z)}{c^{\nu} z(z-w)} \frac{z-z_{\nu, j}}{\psi_{\nu}(z)}=\frac{w \psi_{\nu}\left(c z_{\nu, j}\right)}{c^{\nu} z_{\nu, j}\left(z_{\nu, j}-w\right) \psi_{\nu}^{\prime}\left(z_{\nu, j}\right)}
$$

for $1 \leqq j \leqq N(\nu)$. Since Lemma 3.1 in [10] gives us that, if $\psi_{\nu}\left(z_{0}\right)=0$,

$$
\psi_{\nu+1}\left(z_{0}\right)=-z_{0} \psi_{\nu}^{\prime}\left(z_{0}\right)
$$

we obtain

$$
\operatorname{Res}\left(z_{\nu, j} ; g_{\nu, c}^{w}\right)=-\frac{w \psi_{\nu}\left(c z_{\nu, j}\right)}{c^{\nu}\left(z_{\nu, j}-w\right) \psi_{\nu+1}\left(z_{\nu, j}\right)}
$$

If $z \in D$, it follows from (3.1) that

$$
\frac{K_{\nu}(c z)}{K_{\nu+1}(z)}=\frac{z e^{-(c-1) z} \psi_{\nu}(c z)}{c^{\nu} \psi_{\nu+1}(z)} .
$$

Then $K_{\nu}(c z) / K_{\nu+1}(z)$ is extended to a meromorphic function on $\mathbb{C}$. This implies that $K_{\nu}(c x) / K_{\nu+1}(x)$ can be determined uniquely for $x<0$ with $K_{\nu+1}(x) \neq 0$. From (3.3) and (3.4) we deduce

$$
\operatorname{Res}\left(z_{\nu, j} ; g_{\nu, c}^{w}\right)=-\frac{w e^{(c-1) z_{\nu, j}}}{z_{\nu, j}\left(z_{\nu, j}-w\right)} \frac{K_{\nu}\left(c z_{\nu, j}\right)}{K_{\nu+1}\left(z_{\nu, j}\right)}
$$

and

$$
\Theta(R)=-\frac{1}{c^{\nu}}+e^{(c-1) w} \frac{K_{\nu}(c w)}{K_{\nu}(w)}-\sum_{j=1}^{N(\nu)} \frac{w e^{(c-1) z_{\nu, j}}}{z_{\nu, j}\left(z_{\nu, j}-w\right)} \frac{K_{\nu}\left(c z_{\nu, j}\right)}{K_{\nu+1}\left(z_{\nu, j}\right)} .
$$

$\Theta(R)$ tends to 0 as $R \rightarrow \infty$ since $g_{\nu, c}^{w}(z)=O\left(|z|^{-2}\right)$. Hence we obtain

$$
\frac{K_{\nu}(c w)}{K_{\nu}(w)}= \begin{cases}\frac{e^{-(c-1) w}}{c^{\nu}} & \text { if } \nu=\frac{1}{2} \\ \frac{e^{-(c-1) w}}{c^{\nu}}+\sum_{j=1}^{N(\nu)} \frac{w e^{(c-1)\left(z_{\nu, j}-w\right)}}{z_{\nu, j}\left(z_{\nu, j}-w\right)} \frac{K_{\nu}\left(c z_{\nu, j}\right)}{K_{\nu+1}\left(z_{\nu, j}\right)} & \text { if } \nu \neq \frac{1}{2}\end{cases}
$$

in the case where $\nu-1 / 2$ is a non-negative integer.

We next consider the case where $\nu-1 / 2$ is not an integer and look for a nice expression for $K_{\nu}(c w) / K_{\nu}(w)$ as in (3.5). If $\nu$ is not an integer, it is well known (cf. [18], p. 80) that

$$
K_{\nu}\left(z e^{i m \pi}\right)=e^{-i m \pi \nu} K_{\nu}(z)-i \pi \frac{\sin (m \pi \nu)}{\sin (\pi \nu)} I_{\nu}(z)
$$

for $z \in D$ and $m \in \mathbb{Z}$. When $\nu$ is an integer, we also have

$$
K_{\nu}\left(z e^{i m \pi}\right)=e^{-i m \pi \nu} K_{\nu}(z)-i \pi m(-1)^{(m-1) \nu} I_{\nu}(z)
$$

for $z \in D$ and $m \in \mathbb{Z}$, which is easily seen from

$$
\lim _{\mu \rightarrow n} K_{\mu}(z)=K_{n}(z)
$$

for each integer $n$. Especially, for $z \in D$, we have

$$
\begin{aligned}
& K_{\nu}\left(z e^{i \pi}\right)=e^{-i \pi \nu} K_{\nu}(z)-i \pi I_{\nu}(z), \\
& K_{\nu}\left(z e^{-i \pi}\right)=e^{i \pi \nu} K_{\nu}(z)+i \pi I_{\nu}(z) .
\end{aligned}
$$


It follows from these identities that the function $K_{\nu}(c z) / K_{\nu}(z)$ cannot be extended to a meromorphic function on $\mathbb{C}$. For $z \in D$ let

$$
h_{\nu, c}^{w}(z)=\frac{w e^{(c-1) z}}{z(z-w)} \frac{K_{\nu}(c z)}{K_{\nu}(z)} .
$$

In order to give a formula for $K_{\nu}(c w) / K_{\nu}(w)$ similar to (3.5), we consider the integral of $h_{\nu, c}^{w}$ on a suitable contour. However we cannot adopt a circle as the contour as in (3.2) since $h_{\nu, c}^{w}$ cannot extend to a meromorphic function on $\mathbb{C}$.

Let $\varepsilon$ and $R$ be positive numbers with $2 \varepsilon<R$. We set

$$
\theta_{R, \varepsilon}=\operatorname{Arcsin} \frac{\varepsilon}{R} \text {. }
$$

As a contour, we take the curve $\gamma$ defined by

$$
\begin{aligned}
& \gamma_{0}: z=R e^{i \theta},-\pi+\theta_{R, \varepsilon} \leqq \theta \leqq \pi-\theta_{R, \varepsilon}, \\
& \gamma_{1}: z=x+i \varepsilon,-R \cos \theta_{R, \varepsilon} \leqq x \leqq 0 \\
& \gamma_{2}: z=\varepsilon e^{i \theta},-\pi / 2 \leqq \theta \leqq \pi / 2, \\
& \gamma_{3}: z=x-i \varepsilon,-R \cos \theta_{R, \varepsilon} \leqq x \leqq 0 \\
& \gamma=\gamma_{0}+\gamma_{1}-\gamma_{2}-\gamma_{3} .
\end{aligned}
$$

We take $R$ so large and $\varepsilon$ so small that $w$ and all zeros of $K_{\nu}$ are inside $\gamma$. Then, setting

$$
\Pi(R, \varepsilon)=\frac{1}{2 \pi i} \int_{\gamma} h_{\nu, c}^{w}(z) d z, \quad \Pi_{\ell}=\frac{1}{2 \pi i} \int_{\gamma_{\ell}} h_{\nu, c}^{w}(z) d z
$$

for $0 \leqq \ell \leqq 3$, we have

$$
\Pi(R, \varepsilon)=\Pi_{0}+\Pi_{1}-\Pi_{2}-\Pi_{3} .
$$

The residue theorem yields that, if $N(\nu)=0$,

$$
\Pi(R, \varepsilon)=\operatorname{Res}\left(w ; h_{\nu, c}^{w}\right)
$$

and that, if $N(\nu) \geqq 1$,

$$
\Pi(R, \varepsilon)=\operatorname{Res}\left(w ; h_{\nu, c}^{w}\right)+\sum_{j=1}^{N(\nu)} \operatorname{Res}\left(z_{\nu, j} ; h_{\nu, c}^{w}\right) .
$$

It is obvious that

$$
\operatorname{Res}\left(w ; h_{\nu, c}^{w}\right)=e^{(c-1) w} \frac{K_{\nu}(c w)}{K_{\nu}(w)} .
$$

When $N(\nu) \geqq 1$, by using the formula

$$
z K_{\nu}^{\prime}(z)-\nu K_{\nu}(z)=-z K_{\nu+1}(z)
$$

(cf. [18, p. 29), we can show

$$
\operatorname{Res}\left(z_{\nu, j} ; h_{\nu, c}^{w}\right)=\frac{w e^{(c-1) z_{\nu, j}}}{z_{\nu, j}\left(z_{\nu, j}-w\right)} \frac{K_{\nu}\left(c z_{\nu, j}\right)}{K_{\nu}^{\prime}\left(z_{\nu, j}\right)}=-\frac{w e^{(c-1) z_{\nu, j}}}{z_{\nu, j}\left(z_{\nu, j}-w\right)} \frac{K_{\nu}\left(c z_{\nu, j}\right)}{K_{\nu+1}\left(z_{\nu, j}\right)} .
$$

It follows from (3.8) and (3.9) that, if $N(\nu)=0$,

$$
\Pi(R, \varepsilon)=e^{(c-1) w} \frac{K_{\nu}(c w)}{K_{\nu}(w)}
$$


and that, if $N(\nu) \geqq 1$,

$$
\Pi(R, \varepsilon)=e^{(c-1) w} \frac{K_{\nu}(c w)}{K_{\nu}(w)}-\sum_{j=1}^{N(\nu)} \frac{w e^{(c-1) z_{\nu, j}}}{z_{\nu, j}\left(z_{\nu, j}-w\right)} \frac{K_{\nu}\left(c z_{\nu, j}\right)}{K_{\nu+1}\left(z_{\nu, j}\right)} .
$$

In order to consider the asymptotic behavior of $\Pi(R, \varepsilon)$ as $R \rightarrow \infty$, we need the asymptotic behavior of $K_{\nu}(z)$ as $|z| \rightarrow \infty$. It is known that, if $|\arg z|<3 \pi / 2$,

$$
K_{\nu}(z)=\sqrt{\frac{\pi}{2 z}} e^{-z}\left\{1+O\left(|z|^{-1}\right)\right\}
$$

(cf. [18, p. 202). However it is not sufficient since the error term may not be uniform for $\arg z$. We can improve (3.10) in the following way.

Lemma 3.1. Let $\delta>0$ be given. We have that, for $|\arg z| \leqq 3 \pi / 2-\delta$,

$$
K_{\nu}(z)=\sqrt{\frac{\pi}{2 z}} e^{-z}\left\{1+E_{1}(z)\right\}
$$

where $\left|E_{1}(z)\right| \leqq C_{1} /|z|$ and $C_{1}$ is a positive constant independent of $z$.

Proof. It is known that, for $-\pi+\delta \leqq \arg w \leqq 2 \pi-\delta$,

$$
H_{\nu}^{(1)}(w)=\sqrt{\frac{2}{\pi w}} e^{i(w-\pi \nu / 2-\pi / 4)}\left\{1+E_{2}(w)\right\}
$$

holds for the Hankel function $H_{\nu}^{(1)}$, where $\left|E_{2}(z)\right| \leqq C_{2} /|z|$ and $C_{2}$ is a positive constant which is independent of $z$; see [15], p. 121 and [18, p. 197.

Let $|\arg z| \leqq 3 \pi / 2-\delta$. Then we have $-\pi+\delta \leqq \arg \left(z e^{i \pi / 2}\right) \leqq 2 \pi-\delta$, and thus the formula

$$
K_{\nu}(z)=\frac{1}{2} i \pi e^{i \pi \nu / 2} H_{\nu}^{(1)}\left(z e^{i \pi / 2}\right)
$$

(cf. 18, p. 77) immediately yields the assertion of this lemma.

By virtue of Lemma 3.1, we get

$$
\left|h_{\nu, c}^{w}(z)\right| \leqq \frac{|w|}{\sqrt{c}|z| \cdot|z-w|} \frac{1+C_{1} / c|z|}{1-C_{1} /|z|}
$$

if $z=R e^{i \theta} \in D$ and

$$
\left|\Pi_{0}\right| \leqq \frac{1}{2 \pi} \int_{-\pi+\theta_{R, \varepsilon}}^{\pi-\theta_{R, \varepsilon}}\left|h_{\nu, c}^{w}\left(R e^{i \theta}\right)\right| R d \theta \leqq \frac{|w|}{\sqrt{c}(R-|w|)} \frac{1+C_{1} / c R}{1-C_{1} / R},
$$

which tends to 0 as $R \rightarrow \infty$.

For the integral $\Pi_{1}$, we have

$$
\begin{aligned}
\Pi_{1} & =\frac{w}{2 \pi i} \int_{-R \cos \theta_{R, \varepsilon}}^{0} \frac{e^{(c-1)(x+i \varepsilon)}}{(x+i \varepsilon)(x+i \varepsilon-w)} \frac{K_{\nu}(c(x+i \varepsilon))}{K_{\nu}(x+i \varepsilon)} d x \\
& =\frac{w}{2 \pi i} \int_{0}^{R \cos \theta_{R, \varepsilon}} \frac{e^{(c-1)(-x+i \varepsilon)}}{(x-i \varepsilon)(x-i \varepsilon+w)} \frac{K_{\nu}(c(-x+i \varepsilon))}{K_{\nu}(-x+i \varepsilon)} d x .
\end{aligned}
$$

Then, using (3.6) and writing the right hand side by $\xi_{\nu}$,

$$
\xi_{\nu}(z)=e^{-i \pi \nu} K_{\nu}(z)-i \pi I_{\nu}(z)
$$


we get

$$
\Pi_{1}=\frac{w}{2 \pi i} \int_{0}^{R \cos \theta_{R, \varepsilon}} \frac{e^{-(c-1)(x-i \varepsilon)}}{(x-i \varepsilon)(x-i \varepsilon+w)} \frac{\xi_{\nu}(c(x-i \varepsilon))}{\xi_{\nu}(x-i \varepsilon)} d x .
$$

Hence, letting $\gamma_{1}^{0}$ be the line in $D$ defined by

$$
\gamma_{1}^{0}: z=x-i \varepsilon, 0 \leqq x \leqq R \cos \theta_{R, \varepsilon},
$$

it holds that

$$
\Pi_{1}=\frac{w}{2 \pi i} \int_{\gamma_{1}^{0}} \frac{e^{-(c-1) z}}{z(z+w)} \frac{\xi_{\nu}(c z)}{\xi_{\nu}(z)} d z
$$

Here we define three paths as follows:

$$
\begin{aligned}
& \gamma_{1}^{1}: z=\varepsilon e^{i \theta},-\pi / 2 \leqq \theta \leqq 0 \\
& \gamma_{1}^{2}: z=x, \varepsilon \leqq x \leqq R \\
& \gamma_{1}^{3}: z=R e^{i \theta},-\theta_{R, \varepsilon} \leqq \theta \leqq 0 .
\end{aligned}
$$

Since $w$ is inside $\gamma$, we have that $|\operatorname{Im}(w)|>\varepsilon$ if $\operatorname{Re}(w)<0$. Recall that there is no zero of $K_{\nu}$ on the real axis. Then we may apply the Cauchy integral theorem for the integral on the contour consisting of $\gamma_{1}^{0}, \gamma_{1}^{1}, \gamma_{1}^{2}$ and $\gamma_{1}^{3}$ to obtain

$$
\Pi_{1}=\Pi_{1}^{1}+\Pi_{1}^{2}-\Pi_{1}^{3},
$$

where

$$
\begin{aligned}
& \Pi_{1}^{1}=\frac{w}{2 \pi} \int_{-\pi / 2}^{0} \frac{e^{-(c-1) \varepsilon e^{i \theta}}}{\varepsilon e^{i \theta}+w} \frac{\xi_{\nu}\left(c \varepsilon e^{i \theta}\right)}{\xi_{\nu}\left(\varepsilon e^{i \theta}\right)} d \theta, \\
& \Pi_{1}^{2}=\frac{w}{2 \pi i} \int_{\varepsilon}^{R} \frac{e^{-(c-1) x}}{x(x+w)} \frac{\xi_{\nu}(c x)}{\xi_{\nu}(x)} d x, \\
& \Pi_{1}^{3}=\frac{w}{2 \pi} \int_{-\theta_{R, \varepsilon}}^{0} \frac{e^{-(c-1) R e^{i \theta}}}{R e^{i \theta}+w} \frac{\xi_{\nu}\left(c R e^{i \theta}\right)}{\xi_{\nu}\left(R e^{i \theta}\right)} d \theta .
\end{aligned}
$$

$\Pi_{1}^{3}$ tends to 0 as $R \rightarrow \infty$. In fact, noting that $\xi_{\nu}\left(x e^{i \theta}\right)=K_{\nu}\left(x e^{i(\theta+\pi)}\right)$ holds for $x>0$, we obtain from Lemma 3.1 ,

$$
\frac{\xi_{\nu}\left(c R e^{i \theta}\right)}{\xi_{\nu}\left(\operatorname{Re}^{\theta}\right)}=\frac{1}{\sqrt{c}} e^{-(c-1) R e^{i(\theta+\pi)}} \frac{1+E_{1}\left(c R e^{i(\theta+\pi)}\right)}{1+E_{1}\left(\operatorname{Re}^{i(\theta+\pi)}\right)}
$$

for $|\theta|<\pi / 6$, which yields

$$
\left|e^{-(c-1) R e^{i \theta}} \frac{\xi_{\nu}\left(c R e^{i \theta}\right)}{\xi_{\nu}\left(R e^{\theta}\right)}\right| \leqq \frac{1}{\sqrt{c}} \frac{1+C_{1} / c R}{1-C_{1} / R} \leqq C_{3}
$$

for large $R$ and a positive constant $C_{3}$ which is independent of $R$ and $\theta$. Since $0<\theta_{R, \varepsilon}<\pi / 6$, we see $\Pi_{1}^{3} \rightarrow 0$ as $R \rightarrow \infty$.

Furthermore (3.11) shows that the function $e^{-(c-1) x} \xi_{\nu}(c x) / \xi_{\nu}(x)$ is bounded on $[\varepsilon, \infty)$ and that $\Pi_{1}^{2}$ converges as $R \rightarrow \infty$. Therefore it holds that

$$
\lim _{R \rightarrow \infty} \Pi_{1}=\frac{w}{2 \pi} \int_{-\pi / 2}^{0} \frac{e^{-(c-1) \varepsilon e^{i \theta}}}{\varepsilon e^{i \theta}+w} \frac{\xi_{\nu}\left(c \varepsilon e^{i \theta}\right)}{\xi_{\nu}\left(\varepsilon e^{i \theta}\right)} d \theta+\frac{w}{2 \pi i} \int_{\varepsilon}^{\infty} \frac{e^{-(c-1) x}}{x(x+w)} \frac{\xi_{\nu}(c x)}{\xi_{\nu}(x)} d x .
$$

In the same way, we can show that

$$
\lim _{R \rightarrow \infty}\left(-\Pi_{3}\right)=\frac{w}{2 \pi} \int_{0}^{\pi / 2} \frac{e^{-(c-1) \varepsilon e^{i \theta}}}{\varepsilon e^{i \theta}+w} \frac{\zeta_{\nu}\left(c \varepsilon e^{i \theta}\right)}{\zeta_{\nu}\left(\varepsilon e^{i \theta}\right)} d \theta-\frac{w}{2 \pi i} \int_{\varepsilon}^{\infty} \frac{e^{-(c-1) x}}{x(x+w)} \frac{\zeta_{\nu}(c x)}{\zeta_{\nu}(x)} d x,
$$


where

$$
\zeta_{\nu}(z)=K_{\nu}\left(z e^{-i \pi}\right)=e^{i \pi \nu} K_{\nu}(z)+i \pi I_{\nu}(z)
$$

for $z \in D$ (cf. (3.7)). Note that

$$
\frac{1}{2 \pi i}\left\{\frac{\zeta_{\nu}(c x)}{\zeta_{\nu}(x)}-\frac{\xi_{\nu}(c x)}{\xi_{\nu}(x)}\right\}=\frac{\cos (\pi \nu)\left\{I_{\nu}(c x) K_{\nu}(x)-I_{\nu}(x) K_{\nu}(c x)\right\}}{\left\{K_{\nu}(x)\right\}^{2}+\pi^{2}\left\{I_{\nu}(x)\right\}^{2}+2 \pi \sin (\pi \nu) K_{\nu}(x) I_{\nu}(x)}
$$

and recall that the right hand side is $L_{\nu, c}(x)$. Then we get

$$
\begin{aligned}
\lim _{R \rightarrow \infty} \Pi(R, \varepsilon)= & \frac{w}{2 \pi} \int_{-\pi / 2}^{0} \frac{e^{-(c-1) \varepsilon e^{i \theta}}}{\varepsilon e^{i \theta}+w} \frac{\xi_{\nu}\left(c \varepsilon e^{i \theta}\right)}{\xi_{\nu}\left(\varepsilon e^{i \theta}\right)} d \theta \\
& +\frac{w}{2 \pi} \int_{0}^{\pi / 2} \frac{e^{-(c-1) \varepsilon e^{i \theta}}}{\varepsilon e^{i \theta}+w} \frac{\zeta_{\nu}\left(c \varepsilon e^{i \theta}\right)}{\zeta_{\nu}\left(\varepsilon e^{i \theta}\right)} d \theta \\
& -\frac{w}{2 \pi} \int_{-\pi / 2}^{\pi / 2} \frac{e^{(c-1) \varepsilon e^{i \theta}}}{\varepsilon e^{i \theta}-w} \frac{K_{\nu}\left(c \varepsilon e^{i \theta}\right)}{K_{\nu}\left(\varepsilon e^{i \theta}\right)} d \theta \\
& -\int_{\varepsilon}^{\infty} \frac{w e^{-(c-1) x} L_{\nu, c}(x)}{x(x+w)} d x .
\end{aligned}
$$

We will calculate the limit of each term of (3.12) as $\varepsilon \downarrow 0$.

Lemma 3.2. Let $c>0, \nu \geqq 0$ and $|\theta|<\pi$. We have that

$$
\lim _{\varepsilon \downarrow 0} \frac{K_{\nu}\left(c \varepsilon e^{i \theta}\right)}{K_{\nu}\left(\varepsilon e^{i \theta}\right)}=\lim _{\varepsilon \downarrow 0} \frac{\xi_{\nu}\left(c \varepsilon e^{i \theta}\right)}{\xi_{\nu}\left(\varepsilon e^{i \theta}\right)}=\lim _{\varepsilon \downarrow 0} \frac{\zeta_{\nu}\left(c \varepsilon e^{i \theta}\right)}{\zeta_{\nu}\left(\varepsilon e^{i \theta}\right)}=\frac{1}{c^{\nu}} .
$$

Proof. It is known that

$$
K_{\nu}(z)= \begin{cases}\log \left(\frac{2}{z}\right)\{1+o(1)\} & \text { if } \nu=0, \\ \frac{\Gamma(\nu)}{2}\left(\frac{2}{z}\right)^{\nu}\{1+o(1)\} & \text { if } \nu>0\end{cases}
$$

as $|z| \rightarrow 0$ in $D$; see [15, p. 111 and [18], p. 512. Then, it follows from (3.13) that

$$
\frac{K_{\nu}\left(c \varepsilon e^{i \theta}\right)}{K_{\nu}\left(\varepsilon e^{i \theta}\right)}= \begin{cases}\frac{\log (2 / c \varepsilon)-i \theta}{\log (2 / \varepsilon)-i \theta} \frac{1+o(1)}{1+o(1)} & \text { if } \nu=0, \\ \frac{1}{c^{\nu}} \frac{1+o(1)}{1+o(1)} & \text { if } \nu>0,\end{cases}
$$

which converges to $1 / c^{\nu}$ as $\varepsilon \downarrow 0$. Recall the formula

$$
I_{\nu}(z)=\sum_{n=0}^{\infty} \frac{(z / 2)^{\nu+2 n}}{n ! \Gamma(n+\nu+1)}
$$

for $z \in D$ (cf. [18, p. 77). From (3.13) and (3.14) we deduce that $I_{\nu}\left(x e^{i \theta}\right)$ converges and $K_{\nu}\left(x e^{i \theta}\right)$ tends to infinity as $x \downarrow 0$. This yields that

$$
\frac{\xi_{\nu}\left(c \varepsilon e^{i \theta}\right)}{\xi_{\nu}\left(\varepsilon e^{i \theta}\right)}=\frac{K_{\nu}\left(c \varepsilon e^{i \theta}\right)+O(1)}{K_{\nu}\left(\varepsilon e^{i \theta}\right)+O(1)}=\frac{1}{c^{\nu}}\{1+o(1)\}
$$

as $\varepsilon \downarrow 0$.

We can show

in the same fashion.

$$
\frac{\zeta_{\nu}\left(c \varepsilon e^{i \theta}\right)}{\zeta_{\nu}\left(\varepsilon e^{i \theta}\right)}=\frac{1}{c^{\nu}}\{1+o(1)\}
$$


The first three terms of the right hand side of (3.12) can be calculated easily. Indeed, Lemma 3.2 yields that the first and the second terms converge to $1 / 4 c^{\nu}$ and that the third term converges to $1 / 2 c^{\nu}$. By (3.13) and (3.14), we can easily see

$$
\frac{L_{\nu, c}(x)}{\cos (\pi \nu)}= \begin{cases}\frac{\log c}{(\log x)^{2}}\{1+o(1)\} & \text { if } \nu=0, \\ \frac{c^{\nu}\left(1-c^{-2 \nu}\right) x^{2 \nu}}{2^{2 \nu-1} \Gamma(\nu) \Gamma(\nu+1)}\{1+o(1)\} & \text { if } \nu>0,\end{cases}
$$

as $x \downarrow 0$, which has been noted in [3, p. 29. Hence the last term of the right hand side of (3.12) converges as $\varepsilon \downarrow 0$. Therefore we can conclude

$$
\lim _{\varepsilon \downarrow 0} \lim _{R \rightarrow \infty} \Pi(R, \varepsilon)=\frac{1}{c^{\nu}}-\int_{0}^{\infty} \frac{w e^{-(c-1) x} L_{\nu, c}(x)}{x(x+w)} d x .
$$

Since $K_{\mu}=K_{-\mu}$ for $\mu \geqq 0$, we have that $K_{\nu+1}(z)=K_{|\nu|+1}(z)$ if $z$ is a zero of $K_{\nu}$. Moreover, we can regard $z_{-\nu, j}$ as $z_{\nu, j}$ for $1 \leqq j \leqq N(\nu)$. Therefore we have proven the following.

Theorem 3.3. Let $c>1, \nu \in \mathbb{R}$ and $w$ be a point in $D$ with $K_{\nu}(w) \neq 0$.

(1) If $\nu= \pm 1 / 2$, we have

$$
\frac{K_{\nu}(c w)}{K_{\nu}(w)}=\frac{e^{-(c-1) w}}{c^{|\nu|}} .
$$

(2) If $|\nu|<3 / 2$ and $\nu \neq \pm 1 / 2$, we have

$$
\frac{K_{\nu}(c w)}{K_{\nu}(w)}=\frac{e^{-(c-1) w}}{c^{|\nu|}}-e^{-(c-1) w} \int_{0}^{\infty} \frac{w e^{-(c-1) x} L_{|\nu|, c}(x)}{x(x+w)} d x .
$$

(3) If $\nu-1 / 2$ is an integer and $\nu \neq \pm 1 / 2$,

$$
\frac{K_{\nu}(c w)}{K_{\nu}(w)}=\frac{e^{-(c-1) w}}{c^{|\nu|}}-e^{-(c-1) w} \sum_{j=1}^{N(\nu)} \frac{w e^{(c-1) z_{\nu, j}}}{z_{\nu, j}\left(w-z_{\nu, j}\right)} \frac{K_{\nu}\left(c z_{\nu, j}\right)}{K_{\nu+1}\left(z_{\nu, j}\right)} .
$$

(4) If $\nu-1 / 2$ is not an integer and $|\nu|>3 / 2$,

$$
\begin{aligned}
\frac{K_{\nu}(c w)}{K_{\nu}(w)}=\frac{e^{-(c-1) w}}{c^{|\nu|}} & -e^{-(c-1) w} \sum_{j=1}^{N(\nu)} \frac{w e^{(c-1) z_{\nu, j}}}{z_{\nu, j}\left(w-z_{\nu, j}\right)} \frac{K_{\nu}\left(c z_{\nu, j}\right)}{K_{\nu+1}\left(z_{\nu, j}\right)} \\
& -e^{-(c-1) w} \int_{0}^{\infty} \frac{w e^{-(c-1) x} L_{|\nu|, c}(x)}{x(x+w)} d x .
\end{aligned}
$$

We are ready to complete our proof of Theorem 2.2. We have

$$
\mathcal{L}\left[G_{a, b}^{(\nu)}\right](\lambda)=\frac{1}{\alpha^{\nu}} \frac{K_{\nu}(\alpha \sqrt{\lambda})}{\lambda K_{\nu}(\sqrt{\lambda})}, \quad \alpha=\frac{a}{b}>1 .
$$

We need to derive the inverse Laplace transforms of the following functions:

$$
\begin{aligned}
& p_{1}(\lambda)=\frac{1}{\lambda} e^{-(\alpha-1) \sqrt{\lambda}}, \\
& p_{2}(\lambda ; z)=\frac{1}{\sqrt{\lambda}(\sqrt{\lambda}-z)} e^{-(\alpha-1) \sqrt{\lambda}}, \quad z \in \mathbb{C} .
\end{aligned}
$$


The results may be well known (cf. [16]), but we deduce them from the formula

$$
\int_{0}^{\infty} e^{-\frac{x^{2}}{u^{2}}-\frac{v^{2}}{x^{2}}} d x=\frac{u \sqrt{\pi}}{2} e^{-\frac{2 v}{u}}, \quad u, v>0 .
$$

At first, put

$$
q_{1}(t)=\frac{1}{2 \sqrt{\pi t^{3}}} \int_{\alpha-1}^{\infty} \xi\{\xi-(\alpha-1)\} e^{-\frac{\xi^{2}}{4 t}} d \xi=\frac{1}{\sqrt{\pi t}} \int_{\alpha-1}^{\infty} e^{-\frac{\xi^{2}}{4 t}} d \xi .
$$

Then we get by (3.16)

$$
\begin{aligned}
\int_{0}^{\infty} e^{-\lambda t} q_{1}(t) d t & =\frac{1}{2 \sqrt{\pi}} \int_{\alpha-1}^{\infty} \xi\{\xi-(\alpha-1)\}\left[\int_{0}^{\infty} e^{-\lambda t-\frac{\xi^{2}}{4 t}} t^{-\frac{3}{2}} d t\right] d \xi \\
& =\frac{1}{\sqrt{\pi}} \int_{\alpha-1}^{\infty} \xi\{\xi-(\alpha-1)\}\left[\int_{0}^{\infty} e^{-\frac{\xi^{2} x^{2}}{4}-\frac{\lambda}{x^{2}}} d x\right] d \xi \\
& =\int_{\alpha-1}^{\infty}\{\xi-(\alpha-1)\} e^{-\sqrt{\lambda} \xi} d \xi \\
& =\frac{1}{\lambda} e^{-(\alpha-1) \sqrt{\lambda}}
\end{aligned}
$$

and

$$
\mathcal{L}^{-1}\left[p_{1}\right](t)=q_{1}(t)
$$

Next we put

$$
q_{2}(t ; z)=\frac{1}{\sqrt{\pi t}} \int_{\alpha-1}^{\infty} e^{-\frac{\xi^{2}}{4 t}+z\{\xi-(\alpha-1)\}} d \xi .
$$

Then we obtain from (3.16)

$$
\begin{aligned}
\int_{0}^{\infty} e^{-\lambda t} q_{2}(t ; z) d t & =\frac{1}{\sqrt{\pi}} \int_{\alpha-1}^{\infty} e^{z\{\xi-(\alpha-1)\}}\left[\int_{0}^{\infty} e^{-\lambda t-\frac{\xi^{2}}{4 t}} t^{-\frac{1}{2}} d t\right] d \xi \\
& =\frac{2}{\sqrt{\pi}} \int_{\alpha-1}^{\infty} e^{z\{\xi-(\alpha-1)\}}\left[\int_{0}^{\infty} e^{-\lambda x^{2}-\frac{\xi^{2}}{4 x^{2}}} d x\right] d \xi \\
& =\frac{1}{\sqrt{\lambda}} \int_{\alpha-1}^{\infty} e^{z\{\xi-(\alpha-1)\}-\sqrt{\lambda} \xi} d \xi \\
& =\frac{1}{\sqrt{\lambda}(\sqrt{\lambda}-z)} e^{-(\alpha-1) \sqrt{\lambda}}
\end{aligned}
$$

Hence we get

$$
\mathcal{L}^{-1}\left[p_{2}\right](t)=q_{2}(t ; z)
$$

Now we have shown, for example, for the fourth case where $\nu-1 / 2$ is not an integer and $|\nu|>3 / 2$,

$$
\begin{aligned}
G_{a, b}^{(\nu)}(t)= & \frac{1}{\alpha^{\nu+|\nu|}} \frac{1}{\sqrt{\pi t}} \int_{\alpha-1}^{\infty} e^{-\frac{\xi^{2}}{4 t}} d \xi \\
& -\frac{1}{\alpha^{\nu}} \frac{1}{\sqrt{\pi t}} \sum_{j=1}^{N(\nu)} \frac{K_{\nu}\left(\alpha z_{\nu, j}\right)}{z_{\nu, j} K_{\nu+1}\left(z_{\nu, j}\right)} \int_{\alpha-1}^{\infty} e^{-\frac{\xi^{2}}{4 t}+z_{\nu, j} \xi} d \xi \\
& -\frac{1}{\alpha^{\nu}} \frac{1}{\sqrt{\pi t}} \int_{\alpha-1}^{\infty} e^{-\frac{\xi^{2}}{4 t}}\left[\int_{0}^{\infty} \frac{L_{|\nu|, \alpha}(x)}{x} e^{-x \xi} d x\right] d \xi
\end{aligned}
$$

Finally, a simple change of variables from $\xi$ to $s$ given by $\xi=(a-b) \sqrt{2 t / s}$ gives us the formula in Theorem 2.2(4). The other cases are simpler. 


\section{The taIL PROBABILITy OF THE FIRST Hitting TIME}

As an application of Theorem 2.2, we show the asymptotic behavior of $P\left(\tau_{a, b}^{(\nu)}>\right.$ $t)$ as $t \rightarrow \infty$ when $0<b<a$. In Section 2 we showed when $\nu=0$ by the Tauberian theorem. In [3], it is shown that, if $\nu<0$,

$$
P\left(\tau_{a, b}^{(\nu)}>t\right)=c_{\nu} t^{\nu}\{1+o(1)\}
$$

holds for some constant $c_{\nu}$. It should also be noted that, in [19], Yamazato has discussed the tail probability in a general framework and that some classes of Bessel processes were treated. We give an explicit expression for the constant $c_{\nu}$.

To make the statement clear, we define two constants when $\nu-1 / 2$ is an integer. Put

$$
\sigma_{1}^{(\nu)}=\frac{(a-b)^{2|\nu|}}{2|\nu|} .
$$

Moreover we set $\sigma_{2}^{(\nu)}=0$ if $\nu= \pm 1 / 2$ and

$$
\sigma_{2}^{(\nu)}=b^{2|\nu|}(2|\nu|-1) ! \sum_{j=1}^{N(\nu)} \frac{K_{\nu}\left(a z_{\nu, j} / b\right)}{z_{\nu, j}^{2|\nu|+1} K_{\nu+1}\left(z_{\nu, j}\right)} e^{\frac{z_{\nu, j}(a-b)}{b}} \sum_{k=0}^{2|\nu|-1} \frac{1}{k !}\left\{-\frac{z_{\nu, j}(a-b)}{b}\right\}^{k}
$$

if otherwise.

Theorem 4.1. Let $0<b<a$.

(1) If $\nu=0$,

$$
P\left(\tau_{a, b}^{(0)}>t\right)=\frac{2 \log (a / b)}{\log t}+o\left(\frac{1}{\log t}\right) .
$$

(2) If $\nu>0$ and $\nu-1 / 2$ is an integer,

$$
\begin{aligned}
P\left(\tau_{a, b}^{(\nu)}>t\right)=1-\left(\frac{b}{a}\right)^{2 \nu}+\left(\frac{b}{a}\right)^{2 \nu} \sqrt{\frac{2}{\pi} \frac{(-1 / 2)^{\nu-1 / 2}}{(\nu-1 / 2) !}} & \left\{\sigma_{1}^{(\nu)}+\left(\frac{a}{b}\right)^{\nu} \sigma_{2}^{(\nu)}\right\} \frac{1}{t^{\nu}} \\
& +O\left(\frac{1}{t^{\nu+1}}\right) .
\end{aligned}
$$

(3) If $\nu<0$ and $\nu-1 / 2$ is an integer,

$$
P\left(\tau_{a, b}^{(\nu)}>t\right)=\sqrt{\frac{2}{\pi}} \frac{(-1 / 2)^{-\nu-1 / 2}}{(-\nu-1 / 2) !}\left\{\sigma_{1}^{(\nu)}+\left(\frac{b}{a}\right)^{\nu} \sigma_{2}^{(\nu)}\right\} t^{\nu}+O\left(t^{\nu-1}\right) .
$$

(4) If $\nu>0$ and $\nu-1 / 2$ is not an integer,

$$
P\left(\tau_{a, b}^{(\nu)}>t\right)=1-\left(\frac{b}{a}\right)^{2 \nu}+\left(\frac{b^{3}}{2 a}\right)^{\nu}\left\{\left(\frac{a}{b}\right)^{\nu}-\left(\frac{b}{a}\right)^{\nu}\right\} \frac{1}{\Gamma(1+\nu) t^{\nu}}+o\left(\frac{1}{t^{\nu}}\right) .
$$

(5) If $\nu<0$ and $\nu-1 / 2$ is not an integer,

$$
P\left(\tau_{a, b}^{(\nu)}>t\right)=\left(\frac{2}{a b}\right)^{\nu}\left\{\left(\frac{b}{a}\right)^{\nu}-\left(\frac{a}{b}\right)^{\nu}\right\} \frac{t^{\nu}}{\Gamma(1-\nu)}+o\left(t^{\nu}\right) .
$$

Remark 4.2. It seems that (4) and (5) also hold when $\nu-1 / 2$ is an integer, but we do not pursue the identities.

Before proving this theorem, we give two lemmas. The following is the immediate consequence of Lemma 4.3 as given in [3. We let $m(\nu)$ be the greatest integer which is not larger than $|\nu|-1 / 2$. 
Lemma 4.3. We assume that $|\nu| \geqq 1 / 2$. If $\nu-1 / 2$ is an integer, we have that, for any $0 \leqq m \leqq m(\nu)-1$,

$$
\lim _{t \rightarrow \infty} t^{m+1 / 2} P\left(\tau_{a, b}^{(\nu)}>t\right)=0 .
$$

If $\nu-1 / 2$ is not an integer, we have (4.1) for any $0 \leqq m \leqq m(\nu)$.

For $t>0, \nu \neq 0$ and $z \in \mathbb{C}^{-}$set

$$
\begin{aligned}
& \Psi_{1}(t)=1-\int_{0}^{t} \frac{a-b}{\sqrt{2 \pi s^{3}}} e^{-\frac{(a-b)^{2}}{2 s}} d s \\
& \Psi_{2}(t ; z)=\int_{0}^{t} \frac{a-b}{\sqrt{2 \pi s^{3}}} e^{-\frac{(a-b)^{2}}{2 s}+\frac{z(a-b) \sqrt{t}}{b \sqrt{s}}} d s \\
& \Psi_{3}(t ; \nu)=\int_{0}^{t} \frac{a-b}{\sqrt{2 \pi s^{3}}} e^{-\frac{(a-b)^{2}}{2 s}}\left[\int_{0}^{\infty} \frac{L_{|\nu|, a / b}(x)}{x} e^{-\frac{x(a-b) \sqrt{t}}{b \sqrt{s}}} d x\right] d s .
\end{aligned}
$$

Theorem 2.2 implies that $P\left(\tau_{a, b}^{(\nu)}>t\right)$ is represented by a linear combination of $\Psi_{i}$ 's. Changing variables from $s$ to $u$ by $(a-b) / \sqrt{s}=u$, we have

$$
\begin{aligned}
& \Psi_{1}(t)=1-\sqrt{\frac{2}{\pi}} \int_{(a-b) / \sqrt{t}}^{\infty} e^{-\frac{u^{2}}{2}} d u=\sqrt{\frac{2}{\pi}} \int_{0}^{(a-b) / \sqrt{t}} e^{-\frac{u^{2}}{2}} d u, \\
& \Psi_{2}(t ; z)=\sqrt{\frac{2}{\pi}} \int_{(a-b) / \sqrt{t}}^{\infty} e^{-\frac{u^{2}}{2}+\frac{z \sqrt{t} u}{b}} d u, \\
& \Psi_{3}(t ; \nu)=\sqrt{\frac{2}{\pi}} \cos (\pi \nu) \Psi_{3}^{0}(t ; \nu),
\end{aligned}
$$

where

$$
\Psi_{3}^{0}(t ; \nu)=\int_{(a-b) / \sqrt{t}}^{\infty} e^{-\frac{u^{2}}{2}}\left[\int_{0}^{\infty} \frac{L_{|\nu|, a / b}^{0}(x)}{x} e^{-\frac{x \sqrt{t} u}{b}} d x\right] d u
$$

and $L_{|\nu|, a / b}^{0}(x)=L_{|\nu|, a / b}(x) / \cos (\pi \nu)$. It is obvious that $L_{|\nu|, a / b}^{0}$ is positive on $(0, \infty)$ since $I_{\nu}$ and $K_{\nu}$ is increasing and decreasing on $(0, \infty)$, respectively. For an integer $m$ with $0 \leqq m \leqq m(\nu)$, we set

$$
\begin{aligned}
& \beta_{1}(m)=\frac{(a-b)^{2 m+1}}{2 m+1} \\
& \beta_{2}^{(\nu)}(m)=\sum_{j=1}^{N(\nu)} \frac{K_{\nu}\left(a z_{\nu, j} / b\right)}{z_{\nu, j} K_{\nu+1}\left(z_{\nu, j}\right)} \beta\left(m ; z_{\nu, j}\right), \\
& \beta_{3}^{(\nu)}(m)=(2 m) ! b^{2 m+1} \sum_{k=0}^{2 m} \frac{1}{k !}\left(\frac{a-b}{b}\right)^{k} \int_{0}^{\infty} \frac{L_{|\nu|, a / b}(x)}{x^{2 m-k+2}} e^{-\frac{x(a-b)}{b}} d x,
\end{aligned}
$$

where

$$
\beta(m ; z)=-(2 m) !\left(\frac{b}{z}\right)^{2 m+1} e^{\frac{z(a-b)}{b}} \sum_{k=0}^{2 m} \frac{1}{k !}\left\{-\frac{z(a-b)}{b}\right\}^{k} .
$$

It follows from (3.7) and Lemma 3.1 that

$$
L_{|\nu|, a / b}^{0}(x)=\pi \sqrt{\frac{b}{a}} e^{(a / b-3) x}\{1+o(1)\}
$$


as $x \rightarrow \infty$. Moreover, (3.15) yields that $L_{|\nu|, a / b}^{0}(x) / x^{2 m-k+2}$ is asymptotically equal to a constant multiple of $1 / x^{2 m-k+2-2|\nu|}$ for small $x$. Since

$$
2 m-k+2-2|\nu| \leqq 2 m(\nu)+2-2|\nu|<1,
$$

we have that the following improper integral converges:

$$
\int_{0}^{\infty} \frac{L_{|\nu|, a / b}(x)}{x^{2 m-k+2}} e^{-\frac{x(a-b)}{b}} d x=\cos (\pi \nu) \int_{0}^{\infty} \frac{L_{|\nu|, a / b}^{0}(x)}{x^{2 m-k+2}} e^{-\frac{x(a-b)}{b}} d x .
$$

Lemma 4.4. If $|\nu| \geqq 1 / 2$,

$$
\begin{aligned}
& \Psi_{1}(t)=\sqrt{\frac{2}{\pi}} \sum_{m=0}^{m(\nu)} \frac{(-1 / 2)^{m} \beta_{1}(m)}{m !} \frac{1}{t^{m+1 / 2}}+O\left(\frac{1}{t^{m(\nu)+3 / 2}}\right), \\
& \Psi_{2}(t ; z)=\sqrt{\frac{2}{\pi}} \sum_{m=0}^{m(\nu)} \frac{(-1 / 2)^{m} \beta(m ; z)}{m !} \frac{1}{t^{m+1 / 2}}+O\left(\frac{1}{t^{m(\nu)+3 / 2}}\right) .
\end{aligned}
$$

If $0<|\nu|<1 / 2$,

$$
\Psi_{3}(t ; \nu)=\left(\frac{b^{2}}{2}\right)^{|\nu|}\left\{\left(\frac{a}{b}\right)^{|\nu|}-\left(\frac{b}{a}\right)^{|\nu|}\right\} \frac{1}{\Gamma(1+|\nu|) t^{|\nu|}}+o\left(\frac{1}{t^{|\nu|}}\right) .
$$

If $|\nu|>1 / 2$ and $\nu-1 / 2$ is not an integer,

$$
\begin{aligned}
\Psi_{3}(t ; \nu)= & \sqrt{\frac{2}{\pi}} \sum_{m=0}^{m(\nu)} \frac{(-1 / 2)^{m} \beta_{3}^{(\nu)}(m)}{m !} \frac{1}{t^{m+1 / 2}} \\
& +\left(\frac{b^{2}}{2}\right)^{|\nu|}\left\{\left(\frac{a}{b}\right)^{|\nu|}-\left(\frac{b}{a}\right)^{|\nu|}\right\} \frac{1}{\Gamma(1+|\nu|) t^{|\nu|}} \\
& +o\left(\frac{1}{t^{|\nu|}}\right) .
\end{aligned}
$$

Proof. For $x \geqq 0$ let

$$
P^{(\nu)}(x)=e^{-x^{2} / 2}-\sum_{m=0}^{m(\nu)} \frac{1}{m !}\left(-\frac{x^{2}}{2}\right)^{m}
$$

Note that

Hence we have

$$
\left|P^{(\nu)}(x)\right| \leqq \frac{x^{2 m(\nu)+2}}{2^{m(\nu)+1}\{m(\nu)+1\} !}
$$

$$
\Psi_{1}(t)=\sqrt{\frac{2}{\pi}} \sum_{m=0}^{m(\nu)} \frac{1}{m !}\left(-\frac{1}{2}\right)^{m} \int_{0}^{(a-b) / \sqrt{t}} u^{2 m} d u+\sqrt{\frac{2}{\pi}} \int_{0}^{(a-b) / \sqrt{t}} P^{(\nu)}(u) d u,
$$

which implies (4.2). Similarly, by the formula

$$
\int_{\beta}^{\infty} x^{n} e^{-\mu x} d x=e^{-\beta \mu} \sum_{k=0}^{n} \frac{n !}{k !} \frac{\beta^{k}}{\mu^{n-k+1}}
$$

for $\beta>0$ and $\operatorname{Re}(\mu)>0$ (cf. [8], p. 340), we immediately get (4.3). 
For $|\nu|>1 / 2$ and if $\nu-1 / 2$ is not an integer, we have

$$
\begin{aligned}
\Psi_{3}^{0}(t ; \nu)= & \sum_{m=0}^{m(\nu)} \frac{1}{m !}\left(-\frac{1}{2}\right)^{m} \int_{(a-b) / \sqrt{t}}^{\infty} u^{2 m}\left[\int_{0}^{\infty} \frac{L_{|\nu|, a / b}^{0}(x)}{x} e^{-\frac{x \sqrt{t} u}{b}} d x\right] d u \\
& +\int_{(a-b) / \sqrt{t}}^{\infty} P^{(\nu)}(u)\left[\int_{0}^{\infty} \frac{L_{|\nu|, a / b}^{0}(x)}{x} e^{-\frac{x \sqrt{t} u}{b}} d x\right] d u .
\end{aligned}
$$

Then the first term of the right hand side is equal to

$$
\sum_{m=0}^{m(\nu)} \frac{(-1 / 2)^{m} \beta_{3}^{(\nu)}(m)}{\cos (\pi \nu) m !} \frac{1}{t^{m+1 / 2}}
$$

since

$$
\int_{(a-b) / \sqrt{t}}^{\infty} u^{2 m}\left[\int_{0}^{\infty} \frac{L_{|\nu|, a / b}^{0}(x)}{x} e^{-\frac{x \sqrt{t} u}{b}} d x\right] d u=\frac{\beta_{3}^{(\nu)}(m)}{t^{m+1 / 2}},
$$

which is obtained by the Fubini theorem and (4.6). We set

$$
\widetilde{\Psi}_{3}^{0}(t ; \nu)=\int_{(a-b) / \sqrt{t}}^{\infty} P^{(\nu)}(u)\left[\int_{0}^{\infty} \frac{L_{|\nu|, a / b}^{0}(x)}{x} e^{-\frac{x \sqrt{t} u}{b}} d x\right] d u .
$$

Changing variables from $x$ to $y$ by $x \sqrt{t} u / b=y$, we have

$$
\widetilde{\Psi}_{3}^{0}(t ; \nu)=\int_{0}^{\infty} 1_{\left[\frac{a-b}{\sqrt{t}}, \infty\right)}(u) P^{(\nu)}(u)\left[\int_{0}^{\infty} L_{|\nu|, a / b}^{0}\left(\frac{b y}{\sqrt{t} u}\right) \frac{e^{-y}}{y} d y\right] d u,
$$

where $1_{A}$ is the indicator function of $A$. To see the convergence of $t^{|\nu|} \widetilde{\Psi}_{3}^{0}(t ; \nu)$ as $t \rightarrow \infty$, we need to dominate

$$
t^{|\nu|} 1_{\left[\frac{a-b}{\sqrt{t}}, \infty\right)}(u)\left|P^{(\nu)}(u)\right| L_{|\nu|, a / b}^{0}\left(\frac{b y}{\sqrt{t} u}\right) \frac{e^{-y}}{y}
$$

by an integrable function which is independent of $t$. We have that (4.7) is equal to

$$
b^{2|\nu|} 1_{\left[\frac{a-b}{\sqrt{t}}, \infty\right)}(u)\left|P^{(\nu)}(u)\right| \frac{L_{|\nu|, a / b}^{0}(b y / \sqrt{t} u)}{(b y / \sqrt{t} u)^{2|\nu|}} y^{2|\nu|-1} u^{-2|\nu|} e^{-y} .
$$

Since

$$
\frac{L_{|\nu|, a / b}^{0}(x)}{x^{2|\nu|}} e^{-(a / b-3) x}
$$

is bounded on $(0, \infty)$, we have that $(4.8)$ is dominated by a constant multiple of

$$
1_{\left[\frac{a-b}{\sqrt{t}}, \infty\right)}(u)\left|P^{(\nu)}(u)\right| e^{\frac{y(a-3 b)}{\sqrt{t} u}-y} y^{2|\nu|-1} u^{-2|\nu|} .
$$

We have that, if $a \leqq 3 b$,

$$
e^{\frac{y(a-3 b)}{\sqrt{t} u}-y} \leqq e^{-y}
$$

and that, if $a>3 b$,

$$
e^{\frac{y(a-3 b)}{\sqrt{t} u}-y} \leqq e^{\frac{y(a-3 b)}{a-b}-y}=e^{-\frac{2 b}{a-b} y}
$$

for $u \geqq(a-b) / \sqrt{t}$. Let

$$
\kappa=\min \left\{1, \frac{2 b}{a-b}\right\}
$$

and hence (4.9) is bounded by

$$
\left|P^{(\nu)}(u)\right| u^{-2|\nu|} y^{2|\nu|-1} e^{-\kappa y} .
$$


To see that $\left|P^{(\nu)}(u)\right| u^{-2|\nu|}$ is integrable on $(0, \infty)$, we note that

$$
\left|P^{(\nu)}(x)\right| \leqq C_{4} \min \left\{1, x^{2} / 2\right\} x^{2 m(\nu)}
$$

for some constant $C_{4}$. Then we get

$$
\left|P^{(\nu)}(x)\right| u^{-2|\nu|} \leqq \begin{cases}C_{4} u^{2 m(\nu)-2|\nu|+2} & \text { if } 0<u \leqq \sqrt{2} \\ C_{4} u^{2 m(\nu)-2|\nu|} & \text { if } u>\sqrt{2}\end{cases}
$$

Since

$$
\begin{aligned}
& 2 m(\nu)-2|\nu|+2>2\left(|\nu|-\frac{3}{2}\right)-2|\nu|+2>-1, \\
& 2 m(\nu)-2|\nu|<2\left(|\nu|-\frac{1}{2}\right)-2|\nu|<-1
\end{aligned}
$$

we see that the function given by $(4.10)$ is integrable on $(0, \infty) \times(0, \infty)$. Applying the dominated convergence theorem, the Fubini theorem and (3.15), we have that $t^{|\nu|} \widetilde{\Psi}_{3}^{0}(t ; \nu)$ tends to

$$
\frac{b^{2|\nu|}(a / b)^{|\nu|}\left\{1-(a / b)^{-2|\nu|}\right\}}{2^{2|\nu|-1} \Gamma(|\nu|) \Gamma(|\nu|+1)} \int_{0}^{\infty} P^{(\nu)}(u) u^{-2|\nu|} d u \int_{0}^{\infty} e^{-y} y^{2|\nu|-1} d y
$$

as $t \rightarrow \infty$. Since

$$
\int_{0}^{\infty} e^{-y} y^{2|\nu|-1} d y=\Gamma(2|\nu|)=\frac{2^{2|\nu|-1}}{\sqrt{\pi}} \Gamma(|\nu|) \Gamma\left(\frac{1}{2}+|\nu|\right)
$$

(cf. [15, p. 3), (4.11) coincides with

$$
\frac{b^{2|\nu|}}{\sqrt{\pi}}\left\{\left(\frac{a}{b}\right)^{|\nu|}-\left(\frac{a}{b}\right)^{-|\nu|}\right\} \frac{1}{\Gamma(1+|\nu|)} \Gamma\left(\frac{1}{2}+|\nu|\right) \int_{0}^{\infty} P^{(\nu)}(u) u^{-2|\nu|} d u .
$$

Changing variables from $u$ to $v$ by $v=u^{2} / 2$, we have

$$
\int_{0}^{\infty} P^{(\nu)}(u) u^{-2|\nu|} d u=\frac{1}{2^{|\nu|+1 / 2}} \int_{0}^{\infty} \frac{1}{v^{|\nu|+1 / 2}}\left\{e^{-v}-\sum_{m=0}^{m(\nu)} \frac{(-1)^{m}}{m !} v^{m}\right\} d v,
$$

which is equal to

$$
\frac{1}{2^{|\nu|+1 / 2}} \Gamma\left(\frac{1}{2}-|\nu|\right)
$$

(cf. 8], p. 361). The formula

$$
\Gamma\left(\frac{1}{2}+|\nu|\right) \Gamma\left(\frac{1}{2}-|\nu|\right)=\frac{\pi}{\cos (\pi \nu)}
$$

(cf. [15], p. 3) immediately yields

$$
\widetilde{\Psi}_{3}^{0}(t ; \nu)=\sqrt{\frac{\pi}{2}} \frac{1}{\cos (\pi \nu)}\left(\frac{b^{2}}{2}\right)^{|\nu|}\left\{\left(\frac{a}{b}\right)^{|\nu|}-\left(\frac{b}{a}\right)^{|\nu|}\right\} \frac{1}{\Gamma(1+|\nu|) t^{|\nu|}}+o\left(\frac{1}{t^{|\nu|}}\right)
$$

and hence, we have (4.5).

When $0<|\nu|<1 / 2$, it is enough to consider $\Psi_{3}^{0}(t ; \nu)$ directly. We can easily deduce (4.4) in the same way as $\widetilde{\Psi}_{3}^{0}(t ; \nu)$ for $|\nu|>1 / 2$. The calculation is left to the reader. 
We are now ready to prove Theorem 4.1. We only need to show Theorem 4.1 in the case of $\nu \neq 0$.

For simplicity we set $c=b / a$ and

$$
w_{j}^{(\nu)}=\frac{K_{\nu}\left(a z_{\nu, j} / b\right)}{z_{\nu, j} K_{\nu+1}\left(z_{\nu, j}\right)}
$$

for $1 \leqq j \leqq N(\nu)$. We first consider the case when $\nu-1 / 2$ is not an integer and $\nu<0$. In this case, if $\nu<-3 / 2$, Theorem 2.2 gives

$$
P\left(\tau_{a, b}^{(\nu)}>t\right)=\Psi_{1}(t)+c^{\nu} \sum_{j=1}^{N(\nu)} w_{j}^{(\nu)} \Psi_{2}\left(t ; z_{\nu, j}\right)+c^{\nu} \Psi_{3}(t ; \nu) .
$$

Note that $m(\nu)+1 / 2<|\nu|<m(\nu)+3 / 2$. It follows from Lemma 4.4 that

$$
\begin{aligned}
P\left(\tau_{a, b}^{(\nu)}>t\right)= & \sqrt{\frac{2}{\pi}} \sum_{m=0}^{m(\nu)} \frac{(-1 / 2)^{m}}{m ! t^{m+1 / 2}}\left\{\beta_{1}(m)+c^{\nu} \beta_{2}^{(\nu)}(m)+c^{\nu} \beta_{3}^{(\nu)}(m)\right\} \\
& +c^{\nu}\left(\frac{b^{2}}{2}\right)^{-\nu}\left(c^{\nu}-c^{-\nu}\right) \frac{t^{\nu}}{\Gamma(1-\nu)}+o\left(t^{\nu}\right) .
\end{aligned}
$$

By virtue of Lemma 4.3, we have that, for any $0 \leqq m \leqq m(\nu)$,

$$
\beta_{1}(m)+c^{\nu} \beta_{2}^{(\nu)}(m)+c^{\nu} \beta_{3}^{(\nu)}(m)=0 .
$$

This immediately yields

$$
P\left(\tau_{a, b}^{(\nu)}>t\right)=\left(\frac{2}{a b}\right)^{\nu}\left(c^{\nu}-c^{-\nu}\right) \frac{t^{\nu}}{\Gamma(1-\nu)}+o\left(t^{\nu}\right) .
$$

If $-3 / 2<\nu<0$, Theorem 2.2 gives

$$
P\left(\tau_{a, b}^{(\nu)}>t\right)=\Psi_{1}(t)+c^{\nu} \Psi_{3}(t ; \nu) .
$$

In the case of $-3 / 2<\nu<-1 / 2$, we have, by Lemma 4.4 , that

$$
P\left(\tau_{a, b}^{(\nu)}>t\right)=\sqrt{\frac{2}{\pi t}}\left\{\beta_{1}(0)+c^{\nu} \beta_{3}^{(\nu)}(0)\right\}+\left(\frac{2}{a b}\right)^{\nu}\left(c^{\nu}-c^{-\nu}\right) \frac{t^{\nu}}{\Gamma(1-\nu)}+o\left(t^{\nu}\right) .
$$

Lemma 4.3 yields

$$
\beta_{1}(0)+c^{\nu} \beta_{3}^{(\nu)}(0)=0,
$$

and hence we have (4.13). In the case of $-1 / 2<\nu<0$, Lemma 4.4 gives (4.13) since $\Psi_{1}(t)$ is of order $1 / \sqrt{t}$. We therefore obtain Theorem 4.1(5).

We next consider the case when $\nu-1 / 2$ is not an integer and $\nu>0$. If $\nu>3 / 2$, it follows from Theorem 2.2 that

$$
P\left(\tau_{a, b}^{(\nu)}>t\right)=1-c^{2 \nu}+c^{2 \nu} \Psi_{1}(t)+c^{\nu} \sum_{j=1}^{N(\nu)} w_{j}^{(\nu)} \Psi_{2}\left(t ; z_{\nu, j}\right)+c^{\nu} \Psi_{3}(t ; \nu),
$$

which is equal to

$$
\begin{aligned}
1-c^{2 \nu} & +\sqrt{\frac{2}{\pi}} \sum_{m=0}^{m(\nu)} \frac{(-1 / 2)^{m} c^{2 \nu}}{m ! t^{m+1 / 2}}\left\{\beta_{1}(m)+c^{-\nu} \beta_{2}^{(\nu)}(m)+c^{-\nu} \beta_{3}^{(\nu)}(m)\right\} \\
& +c^{\nu}\left(\frac{b^{2}}{2}\right)^{\nu}\left(c^{-\nu}-c^{\nu}\right) \frac{1}{\Gamma(1+\nu) t^{\nu}}+O\left(\frac{1}{t^{\nu}}\right) .
\end{aligned}
$$


We have that (4.12) is equivalent to

$$
\beta_{1}(m)+c^{-\nu} \beta_{2}^{(-\nu)}(m)+c^{-\nu} \beta_{3}^{(-\nu)}(m)=0
$$

for $0 \leqq m \leqq m(\nu)$. Since $z_{-\nu, j}$ is regarded as $z_{\nu, j}$ and $K_{-\nu+1}\left(z_{-\nu, j}\right)=K_{\nu+1}\left(z_{\nu, j}\right)$ for $1 \leqq j \leqq N(\nu)$, we obtain that $\beta_{2}^{(\nu)}(m)=\beta_{2}^{(-\nu)}(m)$. Moreover it is trivial that $\beta_{3}^{(\nu)}(m)=\beta_{3}^{(-\nu)}(m)$, and hence we have that, for $0 \leqq m \leqq m(\nu)$,

$$
\beta_{1}(m)+c^{-\nu} \beta_{2}^{(\nu)}(m)+c^{-\nu} \beta_{3}^{(\nu)}(m)=0 .
$$

This immediately yields

$$
P\left(\tau_{a, b}^{(\nu)}>t\right)=1-c^{2 \nu}+\left(\frac{b^{3}}{2 a}\right)^{\nu}\left(c^{-\nu}-c^{\nu}\right) \frac{1}{\Gamma(1+\nu) t^{\nu}}+O\left(\frac{1}{t^{\nu}}\right) .
$$

In the other cases, (4.14) can be derived in a similar way. We have proven Theorem $4.1(4)$.

We lastly consider the case when $\nu-1 / 2$ is an integer. As in the case when $\nu-1 / 2$ is not an integer, we can deduce the asymptotic behavior for $\nu>0$ from that for $\nu<0$. Hence we shall treat only the case of $\nu<0$. If $\nu \neq-1 / 2$, Theorem 2.2 and Lemma 4.4 give

$$
\begin{aligned}
P\left(\tau_{a, b}^{(\nu)}>t\right) & =\Psi_{1}(t)+c^{\nu} \sum_{j=1}^{N(\nu)} w_{j}^{(\nu)} \Psi_{2}\left(t ; z_{\nu, j}\right) \\
& =\sqrt{\frac{2}{\pi}} \sum_{m=0}^{m(\nu)} \frac{(-1 / 2)^{m}}{m ! t^{m+1 / 2}}\left\{\beta_{1}(m)+c^{\nu} \beta_{2}^{(\nu)}(m)\right\}+O\left(\frac{1}{t^{m(\nu)+3 / 2}}\right) .
\end{aligned}
$$

Note that $m(\nu)=-\nu-1 / 2 \geqq 1$. It follows from Lemma 4.3 that

$$
\begin{aligned}
P\left(\tau_{a, b}^{(\nu)}>t\right) & =\sqrt{\frac{2}{\pi}} \frac{(-1 / 2)^{m(\nu)}}{\{m(\nu)\} ! t^{m(\nu)+1 / 2}}\left\{\beta_{1}(m(\nu))+c^{\nu} \beta_{2}^{(\nu)}(m(\nu))\right\}+O\left(t^{\nu-1}\right) \\
& =\sqrt{\frac{2}{\pi}} \frac{(-1 / 2)^{-\nu-1 / 2}}{(-\nu-1 / 2) !}\left\{\sigma_{1}^{(\nu)}+c^{\nu} \sigma_{2}^{(\nu)}\right\} t^{\nu}+O\left(t^{\nu-1}\right) .
\end{aligned}
$$

Since

$$
P\left(\tau_{a, b}^{(\nu)}>t\right)=\Psi_{1}(t)=\sqrt{\frac{2}{\pi t}}(a-b)+O\left(\frac{1}{t^{3 / 2}}\right)
$$

if $\nu=-1 / 2$, we obtain Theorem 4.1(3). We have proven Theorem 4.1.

\section{ACKNOWLEDGMENT}

The authors would like to thank Professor Michal Ryznar for valuable comments on the first draft of this work and the anonymous referee for useful information concerning the hitting times of the Bessel processes.

\section{REFERENCES}

[1] A. N. Borodin and P. Salminen, Handbook of Brownian Motion, 2nd ed., Birkhäuser, Basel, 2002. MR.1912205 (2003g:60001)

[2] T. Byczkowski, P. Grazyk and A. Stós, Poisson kernels of half-spaces in real hyperbolic space, Rev. Mat. Iberoam. 23 (2007), 85-126. MR2351127 (2008h:60315)

[3] T. Byczkowski and M. Ryznar, Hitting distribution of geometric Brownian motion, Studia Math. 173 (2006), 19-38. MR2204460 (2007e:60082) 
[4] Z. Ciesielski and S. J. Taylor, First passage times and sojourn times for Brownian motion in space and the exact Hausdorff measure of the sample path, Trans. Amer. Math. Soc. 103 (1962), 434-450. MR0143257 (26:816)

[5] A. Erdélyi, W. Magnus, F. Oberhettinger and F. G. Tricomi, Higher Transcendental Functions. Vol. 2, McGraw-Hill, New York, 1953.

[6] W. Feller, An Introduction to Probability Theory and its Applications, Vol. 2, 2nd ed., Wiley, New York, 1971.

[7] R. K. Getoor and M. J. Sharpe, Excursions of Brownian motion and Bessel processes, Z. Wahr. Ver. Gebiete 47 (1979), 83-106. MR521534 (80b:60104)

[8] I. S. Gradshteyn and I. M. Ryzhik, Table of Integrals, Series, and Products, 7th ed., Academic Press, Amsterdam, 2007. MR2360010 (2008g:00005)

[9] Y. Hamana, On the expected volume of the Wiener sausage, J. Math. Soc. Japan 62 (2010), 1113-1136. MR2761916 (2012b:60270)

[10] _ The expected volume and surface area of the Wiener sausage in odd dimensions, Osaka J. Math. 49 (2012), 853-868.

[11] M. G. H. Ismail, Integral representations and complete monotonicity of various quotients of Bessel functions, Canad. J. Math. 29 (1977), 1198-1207. MR0463527 (57:3474)

[12] K. Itô and H. P. McKean, Diffusion Processes and Their Sample Paths, Springer-Verlag, Berlin-New York, 1974. MR0345224(49:9963)

[13] J. T. Kent, Some probabilistic properties of Bessel functions, Ann. Probab. 6 (1978), 760-770. MR0501378 (58:18750)

[14] Eigenvalue expansion for diffusion hitting times, Z. Wahr. Ver. Gebiete 52 (1980), 309-319. MR576891 (81i:60072)

[15] N. N. Lebedev, Special Functions and Their Applications, Dover, New York, 1972. MR0350075 (50:2568)

[16] F. Oberhettinger and L. Badii, Tables of Laplace Transforms, Springer-Verlag, New YorkHeidelberg, 1973. MR0352889 (50:5375)

[17] D. Revuz and M. Yor, Continuous Martingales and Brownian Motion, 3rd ed., SpringerVerlag, Berlin, 1999. MR1725357 (2000h:60050)

[18] G. N. Watson, A Treatise on the Theory of Bessel Functions, Reprinted of 2nd ed., Cambridge University Press, Cambridge, 1995. MR1349110 (96i:33010)

[19] M. Yamazato, Hitting time distributions of single points for 1-dimensional generalized diffusion processes, Nagoya Math. J. 119 (1990), 143-172. MR.1071905 (93c:60119)

Department of Mathematics, Kumamoto University, Kurokami 2-39-1, Kumamoto, JAPAN 860-8555

Department of Physics and Mathematics, Aoyama Gakuin University, Fuchinobe 510-1, SAgamihara, JAPAN 252-5258 